\section{José Juan Bruner (1825-1899): una estrella fugaz en la historia de la psiquiatría chilena}

\author{
JAIME SANTANDER T., PABLO SANTANDER T., JUAN ENRIQUE BERNER G. ${ }^{a}$
}

\section{The contribution of Jose Juan Bruner to Chilean psychiatry}

The contribution of Dr. Bruner to psychology and psychiatry is largely unknown. This is a summary of the ideas proposed in his "Medical-Psychological Monograph" from 1857, that was written after a case of a possibly possessed woman from Santiago. In this work Dr. Bruner discards the spirit-brain duality, proposes a functional morphology of the brain, recognizes the importance of remote history taking when interviewing patients, proposes a theory for self-formation and the risks of self-fragmentation. He proposes that the case of the woman corresponds to a brain disease, opposing the thought of an ovarian and uterine origin. He proposes a hypothesis of the psychogenic origin of the disease, the importance of what happened during dreams and beyond the conscience of the patient. Many of his ideas preceded by decades those of Charcot and Freud, but they have not had a proper recognition.

(Rev Med Chile 2012; 140: 1495-1500).

Key words: Chile; History of Medicine; Psychiatry.

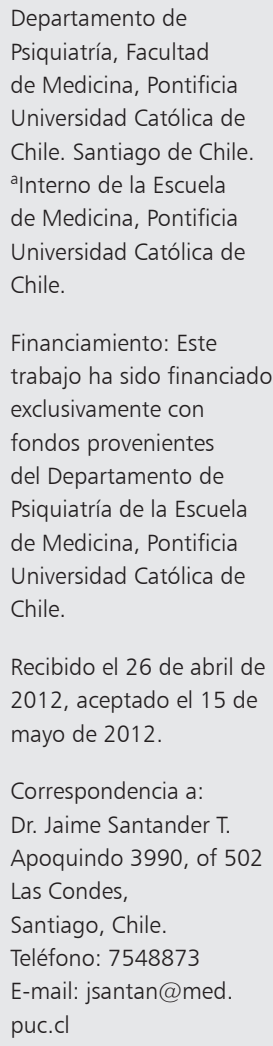

$\mathrm{N}$ umerosas publicaciones han analizado el trabajo y aporte de insignes médicos que, a lo largo de poco más de 150 años, han escrito la historia de la psiquiatría chilena: Manuel Antonio Carmona ${ }^{1,2}$, Carlos Sazié 3 , Ramón Elguero $^{1,4}$, Augusto Orrego Luco ${ }^{1}$, Manuel y Francisco $\mathrm{Beca}^{5}$, Arturo Vivado Orsini ${ }^{6}$, Ignacio Matte Blanco $^{7}$, Juan Marconi ${ }^{7}$ y Armando Roa ${ }^{7}$. En el año 1857 asistimos, a nuestro entender, al nacimiento del pensamiento psiquiátrico en Chile y uno de sus protagonistas no se ha reconocido como tal. Este origen estuvo motivado por el notable caso de la "endemoniada de Santiago" y los extensos trabajos que los doctores Carmona ${ }^{8}$ y Bruner ${ }^{9}$ realizaron al respecto.

El acontecimiento lo resumió Bruner?: "Una joven de 18 años tiene ataques que no se pueden caracterizar. Cae súbitamente con convulsiones, se tuerce, se golpea, se despedaza sin lastimarse, pierde su conocimiento i desarrolla fuerzas extraordinarias; o no cae al suelo, sino que se queda sentada o recostada sin el menor vestijio de sensibilidad, habla cosas obscenas, profiriendo las palabras: monigote, bribón, beata bribona, puta, etc., hasta dicen que habla mal de Dios, llamando a Jesucristo bribón, $i$ a la Virgen, bribona; ve a los sacerdotes que llegan a su cuarto, hasta sabe lo que hacen detrás de ella se exaspera horriblemente cuando le leen cosas sagradas o exorcismos en latín i se tranquiliza cuando se lo manda precisamente un sacerdote en el nombre de Dios pronostica sin equivocarse en un minuto el día i la hora del próximo ataque. Vuelve en sí cuando un sacerdote le pone fin al evanjelio de San Juan i ella aun lo indica para este fin, pero durante la lectura se exaspera en contorsiones horribles, las que se aflojan luego que llega a las palabras: "Y el Verbo fue hecho carne i habitó entre nosotros".

El impacto social de este caso determinó que el Arzobispo de Santiago encargara al Presbítero José Raimundo Zisternas una investigación, para lo cual este último llamó a varios médicos. Seguidamente, aparte de álgidas discusiones en la prensa escrita y revistas de la época, se publicaron los dos interesantísimos textos aludidos que plasmaron, 
alejándose de las causas no naturales que otros defendieron, dos diferentes aproximaciones médicas al caso. Uno de ellos, publicado por Manuel Antonio Carmona, y llamado "Carmen Marín, o la endemoniada de Santiago", compiló los informes rendidos al Arzobispo de Santiago. En este trabajo destacó el análisis comprensivo de la biografía de la paciente, realizado por el mismo Carmona, análisis que llevó a Roa, más de un siglo más tarde, a referirse a este autor como el primer psiquiatra chileno ${ }^{2}$.

En el otro texto publicado a raíz del caso, llamado "La endemoniada de Santiago o el demonio en la naturaleza y la naturaleza del demonio: una monografía médico-psicolójica" (en adelante la "monografía médico-psicolójica", o simplemente, la "monografía”), el doctor José Juan Bruner se acercó al tema de un modo completamente diferente y original. Aunque tanto Carmona como Bruner concordaron en que se trataba de una enfermedad natural y desdeñaron la posibilidad de que la Marín estuviera endemoniada, hicieron un análisis completamente diferente del trasfondo médico de este, determinando Carmona que se trataba "de una enfermedad evidentemente natural que tanto los maestros de la medicina, como el Diccionario de las ciencias médicas, clasifican como perteneciente al orden de las neurosis y cuyo nombre propio es el de histérico confirmado, convulsivo y en tercer grado" originado en ovarios y útero, mientras que Bruner puso todo el problema en una alteración del funcionamiento cerebral ${ }^{9}$, para lo cual hizo un análisis síntoma a síntoma, estableciendo las posibles conexiones con el funcionamiento de dicho órgano.

Bruner, quien no evaluó personalmente a la paciente, pero revisó detalladamente los informes de los facultativos y del presbítero Zisternas, además de hacerse cargo del tema de la "endemoniada", presentó una teoría personal del funcionamiento psicológico normal y patológico, lo que de por si debiera hacerle un lugar en la historia de la psiquiatría nacional, pero lo hizo, además, con momentos de inspiración tal que antecedieron en décadas el surgimiento de conceptos que serían redescubiertos y difundidos por otros autores, varias décadas más tarde.

El tema ha seguido siendo fuente de interés hasta nuestros días como acreditan la publicación, en 1969, de la novela "La endemoniada de Santiago" por Braulio Arenas ${ }^{10}$ y en el año 2010, la reedición del informe completo rendido por Zisternas al Arzobispo, por el periodista Patricio Jara ${ }^{11}$.
El objetivo de esta reseña es reconocer y destacar la obra, los conceptos e ideas en los ámbitos psicológico y de los trastornos mentales, que el Dr. José Juan Bruner instaló en el concierto científico e intelectual criollo.

\section{Antecedentes biográficos}

Hijo de padres alemanes, nació en Varsovia hacia 1825. Emigró muy joven a Berlín, donde estudió medicina. Con 21 años se embarcó en una expedición científica alemana con la que recorrió el mundo, llegando a Chile en 1844, en donde se quedó para hacer estudios naturales. Traía para eso una carta de recomendación de Alexander von Humboldt. Fue profesor en la Universidad de Chile y se dedicó a la investigación y enseñanza de la embriología, histología y química ${ }^{4,12,13}$. Realizó múltiples publicaciones que develaron sus intereses en los ámbitos de la antropología, filosofía y psicología y, gracias al trabajo de Laval, conocemos su participación en problemas relativos a enfermedades epidémicas, como lo ocurrido con el coqueluche en 1851 en La Serena ${ }^{14,15}$ y con la difteria en Santiago en $1876^{16}$. En 1869 concurrió como fundador de la Sociedad Médica de Santiago, en cuya sede lo recuerda un retrato (Figura 1).

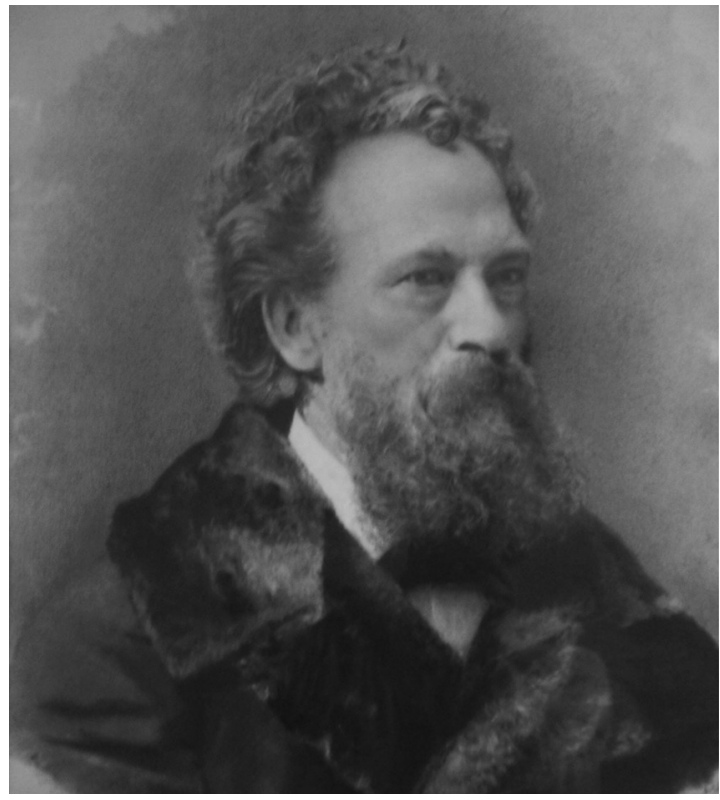

Figura 1. "Dr. José Juan Bruner (1825-1899), Fundador". Retrato existente en la sede de la Sociedad Médica de Santiago. 
José Juan Bruner en la psiquiatría chilena - J. Santander et al

Orrego Luco, en sus "Recuerdos de la Escuela" 4 nos cuenta del que fue su profesor de histología: "tenía esa amabilidad complaciente de los sabios, que siempre gozan cuando pueden prodigar su ciencia" y agrega: "vivía en su gabinete, en medio de sus libros, en su gran laboratorio, haciendo estudios químicos, inclinado sobre un hermoso microscopio. Era una vida misteriosa de trabajo intelectual, solitario y silencioso". Como clínico trabajó inicialmente siguiendo los lineamientos de Hahnemann para luego crear sus propios "procedimientos terapéuticos más innovadores todavía. Él llamó pantobiótica a su sistema curativo", del cual el mismo Orrego Luco confiesa: "nunca pude darme cuenta en qué consistía esa audaz innovación, cuyas ideas fundamentales se presentaban siempre envueltas en los velos del misterio".

Falleció en marzo de 1899, dejando importantes proyectos académicos inconclusos, como su "Historia de la Medicina" y "La Química en sus relaciones con la Biología y la Terapéutica"12.

\section{Publicaciones}

La compilación bibliográfica de Bruner ${ }^{12}$ nos permite tener una visión de la amplitud temática de los trabajos del autor, que comprende poesía, filosofía, medicina y ciencias naturales. Sus trabajos más importantes, así como algunos premios que obtuvo a través de estos ensayos en concursos internacionales se resumen en la Tabla 1.

Nos abocaremos al análisis de su obra de más explícito contenido psicológico y psicopatológico ${ }^{9}$ :

\section{Ideas relevantes de la "Monografía Médico-Psicolójica”"}

En esta "monografía" encontramos un esfuerzo para fundamentar el origen "natural" de la enfermedad de Carmen Marín, pero Bruner toma distancia de Carmona al insistir en que este caso representa un trastorno del funcionamiento "cerebral". Algunas de las ideas más importantes en este conato explicativo se resumen en la Tabla 2:

\section{- Ruptura con dualismo}

Bruner necesitó unir las manifestaciones conductuales y psicológicas al cerebro. Para hacerlo se desligó de la visión dualista imperante en la época: "Para nosotros el alma no es una secreción, sino la enerjia i calidad inmanente del cerebro, así como la visión es la manifestación objetiva de los nervios ópticos, o el magnetismo la fuerza inclita del imán. Para nosotros no existe durante la vida aquel dualismo entre cerebro i espíritu; cada oscilación de una molécula se manifiesta como pensamiento, cada movimiento nutritivo es una sensación, y toda la actividad de nuestra intelijencia es la vibración orgánica de la sustancia cerebral"(pág. 11).

Más adelante, al referirse a las facultades psi-

Tabla 1. Principales publicaciones del doctor José Juan Bruner. Extraída de la obra de F. Bruner ${ }^{12}$

\begin{tabular}{|c|c|c|}
\hline Título & Edición & Comentarios \\
\hline $\begin{array}{l}\text { "De efectu acidorum mineralium in sanguinem } \\
\text { experimentorum et observationum microscopica- } \\
\text { rum pars prima" }\end{array}$ & $\begin{array}{l}\text { 1854, Typis A. Neuenhahni, } \\
\text { Jena, Alemania }\end{array}$ & $\begin{array}{l}\text { Disertación para obtener su grado de } \\
\text { doctor en Medicina, Cirugía y Arte } \\
\text { Obstétrica }\end{array}$ \\
\hline $\begin{array}{l}\text { "El cerebro de los animales i del hombre reducido } \\
\text { a sus tipos fundamentales como símbolos de su } \\
\text { función psicolójica" }\end{array}$ & $\begin{array}{l}\text { 1855, Anales de la Universidad } \\
\text { de Chile, Santiago de Chile }\end{array}$ & $\begin{array}{l}\text { Memoria para obtener el grado de } \\
\text { licenciado en la Universidad de Chile } \\
\text { (convalida estudios) }\end{array}$ \\
\hline $\begin{array}{l}\text { "La endemoniada de Santiago o el demonio en la } \\
\text { naturaleza i la naturaleza del demonio: Monogra- } \\
\text { fía Médico-Psicolójica" }\end{array}$ & $\begin{array}{l}\text { 1857, Imprenta del Ferrocarril, } \\
\text { Santiago de Chile }\end{array}$ & \\
\hline $\begin{array}{l}\text { "El organismo humano enfrente de la naturaleza } \\
\text { circundante" }\end{array}$ & $\begin{array}{l}\text { 1863, Revista "El Criterio Mé- } \\
\text { dico". Imprenta de Vicente y } \\
\text { Lavajos, Madrid, España. }\end{array}$ & $\begin{array}{l}\text { Ensayo ganador del concurso de la } \\
\text { Sociedad Hahnemanniana de Madrid }\end{array}$ \\
\hline "La substancia inmortal del organismo humano" & $\begin{array}{l}\text { 1879, Imprenta Gutemberg, } \\
\text { Santiago de Chile }\end{array}$ & \\
\hline "La experiencia i la especulación" & $\begin{array}{l}\text { 1886, Imprenta Gutemberg, } \\
\text { Santiago de Chile }\end{array}$ & $\begin{array}{l}\text { Medalla de oro en Exposición Mundial } \\
\text { de Barcelona, } 1888\end{array}$ \\
\hline
\end{tabular}




\section{Tabla 2. Ideas relevantes del libro "La endemoniada de Santiago o el demonio en la naturaleza} i la naturaleza del demonio: Monografía Médico-Psicolójica", 1857

\begin{tabular}{|ll|}
\hline Idea & Relevancia \\
$\begin{array}{l}\text { Ruptura con dualismo } \\
\text { cerebro-espíritu }\end{array}$ & $\begin{array}{l}\text { Se atreve a romper con la concepción dualista imperante en América y Europa de la } \\
\text { época }\end{array}$ \\
$\begin{array}{l}\text { Correspondencia } \\
\text { morfológico-funcional }\end{array}$ & $\begin{array}{l}\text { Basado en sus estudios embriológicos y de anatomía comparada propone un modelo que } \\
\text { relaciona desarrollo embrionario, morfología adulta y posible función psicológica. La base } \\
\text { de esta propuesta se encuentra en su ensayo previo, de 1855 }\end{array}$ \\
\hline Constitución del Yo & $\begin{array}{l}\text { Propone una teoría de formación del Yo-mismo y de la personalidad. La primera teoría de } \\
\text { esta naturaleza planteada en Chile }\end{array}$ \\
\hline $\begin{array}{l}\text { Importancia de la historia } \\
\text { remota }\end{array}$ & $\begin{array}{l}\text { Al igual que Carmona comprende la importancia psicológica de conocer la historia del } \\
\text { individuo y sus significados. Inauguran en Chile lo que en Europa sería conocido en la } \\
\text { primera mitad del siglo XX como medicina antropológica }\end{array}$ \\
\hline $\begin{array}{l}\text { Fenómenos inconscientes } \\
\text { y rol de los sueños }\end{array}$ & $\begin{array}{l}\text { Propone la importancia de procesos no conscientes y del rol de los sueños cuando Freud } \\
\text { recién cumplía un año de vida }\end{array}$ \\
$\begin{array}{l}\text { Fenómenos histéricos } \\
\text { de origen cerebral }\end{array}$ & $\begin{array}{l}\text { Antecede en más de dos décadas los trabajos de Charcot que llevaron a la psiquiatría } \\
\text { europea a aceptar la histeria como una enfermedad cerebral }\end{array}$ \\
\hline
\end{tabular}

cológicas (sensación, imaginación, inteligencia y sentimentalidad) las ligó a la materialidad: "Dicha correspondencia funcional está acompañada o más bien producida por una correspondiente acción materialística de los elementos microscópicos de los órganos cerebrales. Función i materialismo se verifican aquí inmediatamente juntos e inseparadamente unidos, en uno i el mismo momento..."(pág. 13).

\section{- Correspondencia morfológica-funcional}

Bruner fue un dedicado investigador y profesor de histología y, fruto de su trabajo, identificó al cerebro como "un sistema de órganos especificos en sí, sin duda no en el sentido huesudo de los frenólogos, sino en el sentido de su evolución morfolójica. A las tres divisiones fundamentales conocidas ya desde Meckel i circunscritas por Carus, he añadido yo una cuarta división" (pp. 12), la cual él mismo había publicado previamente en los "Anales de la Universidad de Chile", en 1855(17). Esta división anatómica es relevante pues sobre ella construyó su teoría psicológica y comienza así: "Como el cerebro se divide en cuatro secciones fundamentales, así también no puede haber más ni menos que cuatro facultades intelectuales, cuatro esferas del alma específicas cada una en sí i distinta cada una de la otra. 1. El órgano protencefálico es el órgano de la Sensación. Dolor, etc. 2. El mesencéfalo es el órgano de la Imajinacion. Imajenes. 3. Los hemisferios son el órgano de la conciencia de sí mismo (Bewusstseyn, consciouness, no conciencia en el sentido relijioso). Ideas. 4. El cerebelo es el órgano de la sentimentalidad (thymos, afectus, Gemueth); de toda la vida interna afectiva del alma: odio, miedo, amor, etc."(pág. 12).

Tras esta presentación en que ligó anatomía cerebral con "facultades" nos introduce en su teoría del Yo, lo cual es relevante para explicar las alteraciones en el comportamiento de Carmen Marín.

\section{- Constitución del Yo y fracturación del Yo}

"La calidad común a todas esas facultades es la subjetividad, i la reflexión de esta subjetividad en sí misma es el Yo. Así cada facultad mental tiene su propio yo mismo, de modo que hai un yo sensitivo, un yo imaginativo, un yo inteligente (conciencia de si mismo) y un yo sentimental" (pág. 12), y agregó que estos Yo fundamentales "se reúnen en la sensación del individuo en un solo i único Yo, en la personalidad..." (pág. 12). "Las enerjías psicolójicas de los cuatro órganos cerebrales se encuentran en una perpetua armonía mutua, en cuanto su independencia relativa lo permite..." (pág. 13), y la pérdida de esta armonía sería causa probable de patología psíquica, lo que abordó en profundidad en el capítulo XVIII, al que llamó: "La fracturación de la personalidad i el cambio del Yo-mismo" (pág. 74), lo que en relación al caso resumió así: "Vemos aquí un elemento puramente relijioso como motivo de su resistencia, $i$ si lo reducimos al yo de donde ha salido, debemos decir que la subjetividad relijiosa de la joven luchaba contra el temor i sus 
José Juan Bruner en la psiquiatría chilena - J. Santander et al

consecuencias que la acosaban. Es esa la lucha de la Carmen contra la Carmen que se emprendió en su alma, una lucha repartida entre dos elementos, ambos pertenecientes a la misma unidad, - el Yo enemistosamente duplicado en medio de su unidad ideal" (pág. 16)

\section{- Importancia de la historia remota}

Bruner reconoció la importancia de la historia del individuo y, pese a las diferencias con Carmona, le reconoce a éste ese mérito: "Lo que en cierto modo nos concilia con el informe en cuestión, son algunas observaciones mui perspicaces que cual relámpagos iluminan momentáneamente el abismo confuso del trabajo entero. Estas observaciones, que se refieren a las reminiscencias que durante el ataque tiene la joven de unas relaciones amorosas con un tal Pascual i un tal Juan..." (pág. 70). Nos explica además: "Muchas veces una enfermedad crónica o aguda no es más que el punto protuberante de la disposición mórbida, que nace de la organización entera, penetrando a su vez todos sus sistemas i procesos. Pero la indole orgánica, que se nutre i edifica del mundo externo desde la primera evolución, tiene su historia, y así la particular historia del individuo es al mismo tiempo la historia de sus enfermedades" (pág. 9). En este contexto conectó los eventos ocurridos a Carmen Marín, a los 12 años, con el origen y desarrollo de la sintomatología que la afectaba y, para explicarnos cómo ocurrió, nos introdujo en el mundo de los sueños: "Pues la joven, después de haber soportado al pié del altar una lucha horrible entre el miedo de quedarse i la vergüenza de huir, dejó la iglesia a las doce de la noche i se acostó.

El sueño es la continuación confusa de la vijilia, y la joven soñó esta misma noche que estaba soñando con el diablo a brazo partido" (pág. 10).

\section{- Clara descripción de fenómenos inconscientes y del rol de los sueños}

Explicó, entonces, que "la imájen del diablo durante su primer sueño es un fenómeno específico que decide para el porvenir el verdadero carácter de los paroxismos; i como esta imájen i no otra fue despertada en ella por lo acaecido en la iglesia, $i$ como no tenía otro efecto pronunciado que el miedo, debemos indagar si acaso se puede encontrar alguna conección interna, psicolójica entre la imájen del diablo i el sentimiento del miedo" (pág. 11), pero aclaró "el endemoniamiento del Yo-mismo se ha desarrollado del sueño, pero no es idéntico al sueño" (pág. 88) y lo explicó partiendo del sueño que dio inicio al cuadro, como el demonio es una simbolización de lo malo y temido, y ese temor arrollador es el que produjo una "inmovilización cataléptica" de ciertas partes del cerebro que desde entonces se alternan en su función, en un inestable equilibrio con otras partes sanas. Vemos aquí una de tantas interpretaciones que hizo Bruner del material psicológico, y todo ese acontecer se escapa al funcionamiento consciente.

\section{- Síntomas histéricos de origen cerebral}

Aunque Bruner no negó que pudiera existir un "verdadero histerismo esencial (sexual)" que "pueda asumir las formas variadas de todas las afecciones nerviosas juntas...” (pág. 68) le pareció que en este caso era evidente el origen cerebral y psicológico de los síntomas. Al hablar de histerismo mencionó explícitamente: "si consideramos el histerismo como una afección primitiva del cerebro, nos queda la inmensa pregunta que contestar, cual es la diferencia que existe entre esta afección cerebral especificamente histérica i las otras semejantes afecciones del sistema encefálico..." (pág. 68).

\section{Comentario final}

Bruner no es un desconocido en la historia de la medicina chilena, y de hecho su aporte fue mencionado por Orrego Luco ${ }^{4}$, Cruz Coke ${ }^{18}$, Laval $^{15,16}$ y otros. Sin embargo, su participación en los ámbitos de la psiquiatría y psicología aparece como marginal. Aunque Roa, en su obra "Demonio y Psiquiatría" transcribió completamente la monografía de Bruner, sólo le dedicó unas pocas palabras. En los albores del pensamiento psiquiátrico en Chile, Carmona ${ }^{1,2}$ discutió las propuestas de Bruner, quien a su vez le contestó ${ }^{8,9}$. Se aludieron y descalificaron sin haber existido la posibilidad de un real diálogo. José Juan Bruner, a raíz del caso de Carmen Marín, no sólo hizo un detallado esfuerzo por mostrar que sus síntomas se debían a causas naturales relativas al cerebro, sino que utilizó el caso para desplegar una teoría del funcionamiento psicológico, sin parangón a esas alturas del desarrollo de la historia de la psiquiatría o la psicología en nuestro país y fue, en varios aspectos, un visionario respecto de una hipótesis acerca del funcionamiento mental.

En efecto, Bruner atribuyó el trastorno de la 
paciente Marín a una alteración en su funcionamiento cerebral y, aunque a la fecha había quienes en Europa habían atribuido los cuadros histéricos al cerebro ${ }^{9}$, aún predominaría por décadas -hasta Charcot- la idea de que se originaban en el sistema genital femenino. En Chile, aunque él no generalizó a la histeria como una enfermedad de origen cerebral, al menos apuntó que, en casos como el de la paciente Marín, se debía a una alteración a ese nivel. Para arribar a esa explicación derribó el dualismo cartesiano imperante y en un discurso claramente materialista atribuyó espíritu y alma al funcionamiento cerebral. Debemos precisar que Bruner no mencionó la palabra "mente" y, al referirse a alma o espíritu, no lo hizo pensando en un componente trascendente de la vida del hombre sino en aquellas facultades que hoy reconocemos como propias del funcionamiento mental.

En su "monografía médico-psicolójica" nos mostró la formación del Yo y los riesgos de una fracturación del mismo. Al igual que Carmona, reconoció la importancia de la historia biográfica del individuo en el desarrollo de sus procesos mórbidos. Puso, además, de manifiesto la importancia de los sueños en los procesos mentales, en un momento en que Freud celebraba su primer cumpleaños. Aunque no habló del "inconsciente", toda su elaboración psicológica requirió de procesos subyacentes que se escapan de la subjetividad consciente del individuo.

En resumen, Bruner se adelantó por décadas a sus contemporáneos en Chile y en otras latitudes pero, sin tener interlocutores ni discípulos (al menos en el terreno de los trastornos mentales) sus ideas innovadoras y rupturistas han parecido marchitarse. El destino del Dr. J. Juan Bruner fue convertirse en una estrella fugaz en nuestra historia psiquiátrica nacional. Sin embargo, dadas su precocidad, audacia y originalidad, bien vale revisar su trabajo e instalarlo en el lugar que le corresponde.

\section{Referencias}

1. Roa A. Demonio y Psiquiatría. Santiago, Chile: Editorial Andrés Bello, 1974.

2. Roa A. Un caso de posesión demoniaca en el Chile del siglo XIX: su trascendencia para el desarrollo de la psiquiatría chilena. Acta Psiquiatr Psicol Am Lat 1991; 37 (1): 78-9.

3. Escobar E. Carlos Sazié Heredia, segundo profesor de neurología y enfermedades mentales en Chile (18521921). Rev Chil Neuro-Psiquiat 2001; 39 (2): 165-6.

4. Orrego Luco A. Recuerdos de la Escuela. Buenos Aires, Argentina: Editorial Francisco de Aguirre, 1976, tercera edición.

5. Escobar E. Los Beca en la psiquiatría chilena. Rev Med Chile 2000; 128: 827-32.

6. Escobar E. Arturo Vivado Orsini (1894-1949) y sus aportes a la psiquiatría chilena. Rev Chil Neuro-Psiquiat 2006; 44 (1): 54-6.

7. Ojeda C. Ignacio Matte Blanco, Armando Roa Rebolledo y Juan Marconi Tassara: tres creadores en la psiquiatría chilena. Rev Chil Neuro-Psiquiat 2001; 39 (3): 183-93.

8. Carmona, MA. Carmen Marín, o la endemoniada de Santiago. Valparaíso, Chile: Imprenta y Librería del Mercurio, 1857.

9. Bruner JJ. La endemoniada de Santiago o el demonio en la naturaleza i la naturaleza del demonio: una monografía médico-psicolójica. Santiago, Chile: Imprenta del Ferrocarril, 1857.

10. Arenas B. La endemoniada de Santiago. Caracas, Venezuela: Monte Avila Editores, 1969.

11. Jara P. La endemoniada de Santiago. Santiago, Chile: Ediciones B (Vergara), 2010.

12. Bruner F. Biblografía del Dr. J. Juan Bruner. Santiago, Chile: Imprenta Universitaria, 1916.

13. Cubillos L. Epistolario de Alexander von Humboldt con personajes vinculados a Chile. Revista Universum 2002; 17: 37-52.

14. Bruner JJ. Sobre la epidemia de 1851 en La Serena por el Dr Brunner. Memoria trabajada en octubre de 1853, i presentada a la Facultad de Medicina en 1854. Anales de la Universidad de Chile 1854; 39-42.

15. Laval E. Contribución a la historia de la coqueluche en Chile. Rev Chil Infectol 2010; 27 (4): 351-5.

16. Laval E. Difteria en Chile. Rev Chil Infectol, edición aniversario 2003; 42.

17. Bruner J. El cerebro de los animales i del hombre reducido a sus tipos fundamentales como símbolos de su función psicolójica. Anales de la Universidad de Chile 1855; 674-84.

18. Cruz-Coke. Historia de la Medicina Chilena. Santiago, Chile: Editorial Andrés Bello, 1995. 